\title{
Does serological evidence of prior infection with SARS-CoV-2 indicate immunity
}

\begin{abstract}
As the novel coronavirus 2019 (SARS-CoV-2) pandemic has developed worldwide, epidemiologists, infectious disease specialists, and public health officials, among other invested parties, have grappled with the question of to what degree viral re-infection and/ or re-activation are issues of concern. ${ }^{1,2}$ SARS-CoV-2 infected patients tend to develop an antibody response, and there are hopes that this represents immunity on the behalf of serologically positive patients. ${ }^{3,4}$ Here we present two cases where a patients presented with symptomatic SARS-CoV-2 infection while simultaneously exhibiting positive antibody tests. One of the goals of SARS-CoV-2 antibody tests is to determine who is still at risk of infection. ${ }^{5}$ However it is still not clear if infection confers immunity ${ }^{6,7}$ There has also been question as to the reliability and accuracy of these tests. ${ }^{8}$ These cases demonstrate that there are still many unknowns with regards to SARS-CoV-2 testing, particularly with regards to immunity and vaccine development. ${ }^{9-11 .}$
\end{abstract}

Keywords: coronavirus, COVID19, severe acute respiratory syndrome coronavirus 2, pandemics, immunity, immunization
Volume 8 Issue 3 - 2020

\author{
Adan Bashir, Joshua F Potter, Eric Sin \\ Division of infectious diseases, Stony Brook Southampton \\ Hospital, USA
}

\begin{abstract}
Correspondence: Eric Sin, Division of infectious diseases, Stony Brook Southampton Hospital, 240 Meeting House Lane, Southampton, NY, I 1 968, United States, Tel (631) 283-2100,
\end{abstract} Fax (63I) 283-8870, Email eric.sin@stonybrookmedicine.edu

Received: July 15, 2020 | Published: July 30, 2020

\section{Introduction}

As the novel coronavirus 2019 (SARS-CoV-2) pandemic has developed worldwide, epidemiologists, infectious disease specialists, and public health officials, among other invested parties, have grappled with the question of to what degree viral re-infection and/or re-activation are issues of concern. ${ }^{1,2}$ SARS-CoV-2 infected patients tend to develop an antibody response, and there are hopes that this represents immunity on the behalf of serologically positive patients. ${ }^{3,4}$ Here we present two cases where patients developed symptomatic SARS-CoV-2 infection as evidenced by symptoms consistent with COVID19 requiring hospitalization and confirmed by a positive PCR nasopharyngeal swab despite having a positive antibody test as well.

\section{Patient A}

In March 2020, an 83-year-old man from Brooklyn, New York, went to a walk in clinic where he had a SARS-CoV-2 real-time reverse-transcriptase polymerase chain reaction (RT PCR) done for screening. He was asymptomatic, but his screening test came back positive. This man lived with his wife, who had recently died shortly thereafter due to complications from SARS-CoV-2 infection. Eight weeks later, he presented to the emergency room at our institution with chief complaints of dyspnea and cough. His oxygen saturation was $99 \%$ on 3 liters nasal cannula, and his temperature was $97.6 \mathrm{~F}$. Chest X-ray was suggestive of multifocal pneumonia consistent with viral illness, and he was started on ceftriaxone and doxycycline. Labs were notable for a procalcitonin of $0.15 \mathrm{ng} / \mathrm{mL}$, ferritin of $931.1 \mathrm{ng} /$ $\mathrm{mL}$, and a D-Dimer of $706 \mathrm{ng} / \mathrm{mL}$. Blood, urine and sputum cultures were drawn and were ultimately negative. SARS-CoV-2 RT PCR (Xpert Xpress) and antibody test (Abbott SARS COV2 COVID19 IGG Antibody) were ordered. Both his PCR and antibody tests came back positive. Apart from coronary artery disease and one cardiac stent, he had no chronic medical conditions. While hospitalized, his respiratory status continued to worsen and he was placed on a nonrebreather mask, followed by high flow nasal cannula. Despite this, his oxygen saturation decreased to the low $50 \%$, and he was placed on mechanical ventilation. He was given convalescent plasma and a five day course of remdesevir. Throughout his hospital stay he required increasing vasopressor support and developed increasing oxygen requirements. He was intubated for 25 days until his children decided to transition him to comfort care. He died shortly after a terminal extubation.

\section{Patient B}

In April a 61-years-old man went to the emergency with dyspnea and a cough. His temperature was $99.3 \mathrm{~F}$ and he was saturating $82 \%$ on room air which improved to $95 \%$ on four liters nasal cannula. On imaging his chest $\mathrm{x}$-ray showed bilateral hazy opacities. SARSCoV-2 RT PCR (Xpert Xpress) was positive. A trial course of hydroxychloroquine, thiamine and zine was given for five days. He was managed in the intensive care unit on high flow nasal cannula and after 21 days of hospitalization he was discharged. His comorbid conditions include type 2 diabetes on metformin with a recent hemoglobin $\mathrm{A} 1 \mathrm{C}$ of $9.2 \%$. 42 days after his initial presentation to the hospital he returned to his local emergency room with dyspnea. Chest $\mathrm{x}$-ray showed bilateral pleural effusions and a thoracentesis was done where 1,900 milliliters of fluid were drained. SARS-CoV-2 RT PCR (Xpert Xpress) was negative and antibody test (Abbott SARS COV2 COVID19 IGG Antibody) was positive. The patient felt better and left against medical advice. Thirteen days after his second presentation he returned to the emergency room with cough, dyspnea and shortness of breath. The man's temperature was $97.9 \mathrm{~F}$ and his oxygen saturation was $96 \%$ on room air. Chest x-ray showed bilateral hazy opacities and moderate bilateral plural effusions. A repeat SARS-CoV-2 RT PCR (Xpert Xpress) was positive. On trans-thoracic echocardiogram his ejection fraction was $65 \%$ with normal left ventricular systolic function. His respiratory status continued to decline and the patient suffered a cardiac arrest. There was return of circulation after two rounds of chest compressions and epinephrine. He was given convalescent plasma and a five day course of remdesevir. The patient was extubated after six days and a repeat SARS-CoV-2 RT PCR (Xpert Xpress) was negative. After seven more days in the hospital he was discharged home. 


\section{Discussion}

Patient A was exposed to and tested positive for the SARSCoV-2 virus as supported by PCR testing in March 2020. Although asymptomatic at the time of his initial testing, he later subsequently developed cough and respiratory distress approximately eight weeks later, prompting him to present to the hospital as detailed above. Here, he tested positive for both SARS-CoV-2 RT PCR and IgG antibody tests. His respiratory status continued to decline in a pattern consistent with active SARS-CoV-2 infection eventually succumbing to his illness. Patient B was PCR positive for the SARS-CoV-2 virus and was symptomatic. He recovered during his initial hospitalization and was discharged. 42 days after presentation he was SARS-CoV-2 PCR negative and antibody positive. 13 days later he again developed respiratory symptoms and hospitalized. Subsequent SARS-CoV-2 RT PCR was again positive. Two concerns are raised from these cases: 1) Are the repeat positive SARS-CoV-2 PCR tests representative of a reinfection or reactivation, and 2) If so, what does that imply about the degree of immunity, if any, that SARS-CoV-2 antibodies confer from reinfection? The median IgG seroconversion time was found to be 14 days with titers that plateau within 6 days after seroconversion. ${ }^{12-14}$ which has been useful in contact tracing. ${ }^{15}$ One of the goals of SARSCoV-2 antibody tests is to determine who is still at risk of infection9. However it is still not clear if infection confers immunity. ${ }^{6,7}$ There has also been question as to the reliability and accuracy of these tests. ${ }^{8}$ These cases demonstrate that there are still many unknowns with regards to SARS-CoV-2 testing, particularly with regards to immunity after infection and its implications in vaccine. ${ }^{13-15}$

\section{Acknowledgments}

None.

\section{Conflicts of interest}

None.

\section{References}

1. Alvarez-Moreno CA, Rodríguez-Morales AJ. Testing Dilemmas: Post negative, positive SARS-CoV-2 RT-PCR - is it a reinfection? Travel Med Infect Dis. 2020;101743.
2. Ravioli S, Ochsner H, Lindner G. Reactivation of COVID-19 pneumonia: A report of two cases. $J$ Infect. 2020;7;81(2):e72-e73.

3. Zhao J, Yuan Q, Wang H, et al. Antibody responses to SARS-CoV-2 in patients of novel coronavirus disease 2019. Clin Infect Dis. 2020;ciaa344.

4. Petherick A. Developing antibody tests for SARS-CoV-2. Lancet. 2020;395(10230):1101-1102.

5. Zhao J, Yuan Q, Wang H, et al. Antibody responses to SARS-CoV-2 in patients of novel coronavirus disease 2019. Clin Infect Dis. 2020;ciaa344.

6. Krammer F, Simon V. Serology assays to manage COVID-19. Science. 2020;368(6495):1060-1061.

7. Vabret N, Britton GJ, Gruber C, et al. Immunology of COVID-19: Current State of the Science. Immunity. 2020;52(6):910-941.

8. Mallapaty S. Will antibody tests for the coronavirus really change everything? Nature. 2020;580(7805):571-572.

9. Espejo AP, Akgun Y, Al Mana AF, et al. Review of Current Advances in Serologic Testing for COVID-19. Am J Clin Pathol. 2020;aqaa112.

10. Li Z, Yi Y, Luo X, et al. Development and clinical application of a rapid IgM-IgG combined antibody test for SARS-CoV-2 infection diagnosis. $J$ Med Virol. 2020.

11. Infantino M, Damiani A, Gobbi FL, et al. Serological Assays for SARSCoV-2 Infectious Disease: Benefits, Limitations and Perspectives. Isr Med Assoc J. 2020;22(4):203-210.

12. Qu J, Wu C, Li X, et al. Profile of IgG and IgM antibodies against severe acute respiratory syndrome coronavirus 2 (SARS-CoV-2). Clin Infect Dis. 2020; ciaa489.

13. Caturegli G, Materi J, Howard BM, et al. Clinical Validity of Serum Antibodies to SARS-CoV-2: A Case-Control Study. Ann Intern Med. 2020;M20-2889.

14. Long QX, Liu BZ, Deng HJ, et al. Antibody responses to SARS-CoV-2 in patients with COVID-19. Nat Med. 2020;26(6):845-848.

15. Zhang G, Nie S, Zhang Z, et al. Longitudinal Change of Severe Acute Respiratory Syndrome Coronavirus 2 Antibodies in Patients with Coronavirus Disease 2019. J Infect Dis. 2020;222(2):183-188. 